\title{
Case of bilateral iatrogenic medial canthal webbing treated with full thickness skin grafts
}

\begin{abstract}
Epicanthal webbing can result from both traumatic and iatrogenic injuries. The latter is usually related to skin incisions required to gain external access to the ethmoidal and frontal sinuses or more rarely to the lacrimal sac. Surgical management traditionally involves a Z-plasty or V-to-Y plasty of the medial canthus to increase the length of the vertical scar, thus releasing the web contraction.

These methods, however, may present challenging limitations when the webbing is too close to the medial canthus, since the potential length of one of the plasty limb is greatly restricted by the vicinity of the eye. This case report exemplifies such a scenario, which required an alternative solution with full thickness skin grafts (FTSG)
\end{abstract}

Keywords: medial canthus, webbing, full thickness skin grafts
Volume 4 Issue 5 - 2016

\author{
Sandro Chiti-Batelli,' Viktoria \\ Grammatopoulou ${ }^{2}$ \\ 'Rhinologist, Facial Plastic and ENT Locum Consultant, Royal \\ Surrey County Hospital, UK \\ 2ENT Specialty Doctor, Royal Surrey County Hospital, UK
}

Correspondence: Sandro Chiti-Batelli, Rhinologist, Facial Plastic and ENT Locum Consultant, Ear, Nose and Throat Department, Egerton Road, Guildford, Surrey, GU2 7XX, UK, Email sachb|62@gmail.com

Received: August 06, 2016 | Published: October 03, 2016

\section{Case presentation}

A 37-year-old female sustained a domestic facial injury after falling from the stairs. The deep horizontal skin laceration that resulted from the trauma extended over the nasal dorsum, approximately $5 \mathrm{~mm}$ from each medial canthus. The cutaneous defect was debrided from the crushed nonviable tissue and sutured by a junior doctor in the Accident and Emergency Department. Unfortunately the wound contracted over the following two weeks and the patient presented again to the hospital lamenting a clear cosmetic iatrogenic webbing deformity over the medial canthi.

Two months later she was reassessed in order to allow some more time for scar maturation and to plan the surgical treatment (Figure $1,2 \mathrm{~A} \& 2 \mathrm{~B})$. Ideally, it would have been preferable to delay the operation for a longer period of time, but the intense emotional distress experienced by the patient in view of the approaching wedding day, scheduled for the following month, weakened our resistance and it was agreed to proceed with the operation within the next few days.

It was felt that the scars were too close to the medial canthi for a Z- or V-to-Y-plasty and an alternative, although uncharted solution for the specific defect was chosen. Two FTSG were harvested under general anesthesia from the left post-auricular matching colored area. The scars were released with a horizontal incision of approximately $7 \mathrm{~mm}$ and the skin undermined (Figure 3A\&3B). The fat was carefully removed from both grafts and they were fashioned to match the defects and then sutured and secured with cotton bolsters in the usual manner. Fortunately the procedure was successful (Figure 4,5A\&5B) and almost more importantly warded off the cancelling of the wedding.

\section{Discussion}

Epicanthal folds are a natural feature in Asian populations, whereas in Caucasian individuals are typically a result of trauma or surgery. Fronto-ethmoidal external approaches and more rarely external DCR and blepharoplasty represent the commonest iatrogenic causes of medial canthal webbing. The scars usually occur when the incisions are carried too medially and the skin bridges the supero-medial hollow of the upper lid in a straight line. In some cases early recognition and aggressive massage can result in better aesthetic results by improving the circulation. Persistent scars are traditionally treated either with a Z-, W- or a V- to-Y-plasty. ${ }^{1}$ Rhomboid and bilobed flaps have also been described. ${ }^{2-4}$

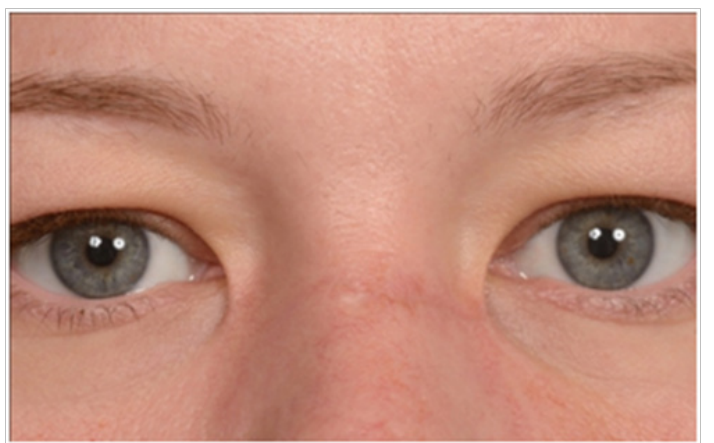

Figure I Pre-operative frontal view of bilateralepicanthal webbing and scar extended over the nasal dorsum as a result of cosmetic iatrogenic deformity.

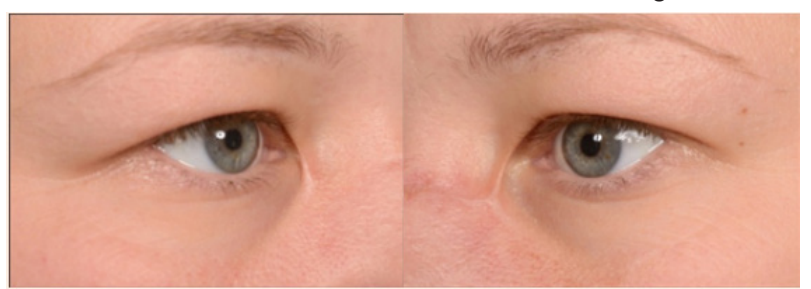

Figure 2A\&B Pre-operative three quaters views (A-right, B-left) of medial epicanthal webbing and nasal dorsum scar.

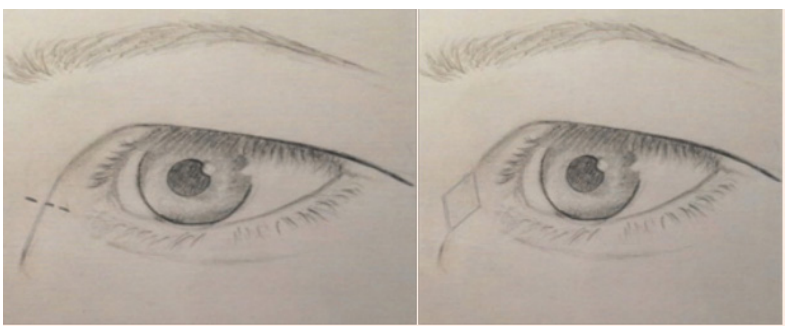

Figure 3A\&B Drawings showing the surgical technique :A-horizontal dotted line indicates there leasing incision over the Canthal webbing. B-Rhomboid shape are as how sunder mined soft tissue and graft placement. 


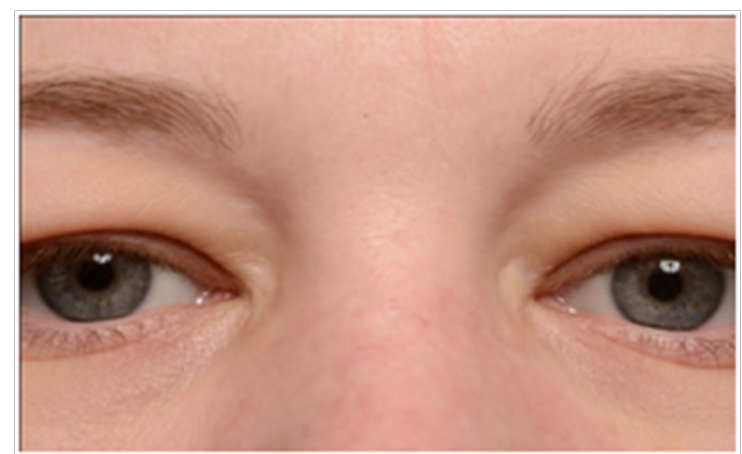

Figure 4 Six month's post-operative frontal view after the scar was released and FTSG were harvested from left post-auricular area. Note the absence of epicanthal webbing and prominent scar of nasal dorsum.

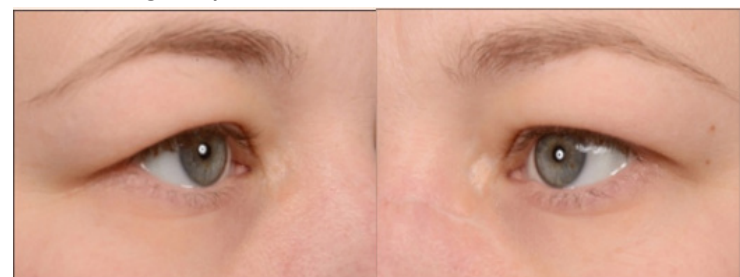

Figure 5A\&B Six month's post-operative frontal view after the scar was released and FTSG were harvested from left post-auricular area. Note the absence of epicanthal webbing and prominent scar of nasal dorsum.

In those circumstances, where the scars appear too close to the medial canthus, skin grafting is a possible alternative approach. Although FTSG have been used to reconstruct the medial canthus following the tumor excision, no other reports to our knowledge, have described them as a specific treatment for medial canthal webbing. ${ }^{5}$ Horizontal division of the web and generous undermining facilitates placement of the graft, which should be quite redundant in order to compensate for scar contraction.

Choice of the donor site is also very important. The post-and pre-auricular as well as the eyelid skin region are more suitable for thickness and color matching. Careful removal of the fat tissue and smoking avoidance are quite crucial to maximize the restoration of blood supply to the skin and to create the optimal conditions for the graft to take in such a delicate area.

\section{Conclusion}

This case report suggests that FTSG should be considered as an additional surgical method to correct both traumatic and iatrogenic epicanthal webbing, especially when the scars are in close vicinity to the eye.

\section{Acknowledgements}

We would like to thank Guilia Vera Chiti-Batelli for her great graphic support in illustrating the surgical steps.

\section{Conflicts of interest}

Authoer declares there are no conflicts of interest.

\section{Funding}

None.

\section{References}

1. Patrinely JR, Marines HM, Anderson RL. Skin flaps in periorbital reconstruction. Surv Ophthalmol. 1987;31(4):249-261.

2. Ng SG, Inkster CF, Leatherbarrow B. The rhomboid flap in medial canthal reconstruction. Br J Ophthalmol. 2001;85(5):556-559.

3. Shotton FT. Optimal closure of medial canthal surgical defects with rhomboid flaps: "rules of thumb" for flap and rhomboid defect orientations. Ophthalmic Surgery, Laser and Imaging Retina. 1983;14(1):46-52.

4. Sullivan TJ, Bray LC. The bilobed flap in medial canthal reconstruction. Aust N Z J Ophthalmol. 1995;23(1):42-48.

5. Lodde JP, Valentin S. Reconstruction of the medial canthus in oncology. Apropos of 105 cases over 9 years. Ann Chir Plast Esthet. 1991;36(5):371-376. 